\title{
Les infections entériques sont-elles transmises sexuellement en Colombie-Britannique?
}

\author{
Narayan $\mathrm{S}^{1 *}$, Galanis $\mathrm{E}^{2}$, Groupe de la Colombie-Britannique sur les infections entériques \\ transmises sexuellement ${ }^{3}$
}

\section{Résumé}

Contexte : Parfois, les infections entériques peuvent se transmettre sexuellement, en particulier chez les personnes ayant des relations sexuelles orales et anales. Bien que des éclosions d'infections entériques aient été signalées chez les hommes ayant des relations sexuelles avec d'autres hommes (HARSAH) en Colombie-Britannique, l'épidémiologie des infections entériques transmises sexuellement n'a jamais été évaluée.

Objectif : Décrire l'épidémiologie des infections entériques en Colombie-Britannique afin de déterminer si une transmission sexuelle peut se produire.

Méthodologie : Une analyse descriptive de tous les cas signalés de shigellose, d'amibiase et de giardiase en Colombie-Britannique de 2003 à 2012 a été effectuée.

Résultats : Pour la shigellose et l'amibiase, il y avait un rapport élevé hommes-femmes et un rapport d'infection élevé chez les hommes âgés de 20 à 59 ans, par rapport à tous les autres groupes d'âge et de sexe. En outre, pour la shigellose, les hommes adultes étaient plus susceptibles de contracter la maladie localement que les femmes (RR : 1,9; IC: 1,7 à 2,4).

Conclusion : L'analyse laisse entendre que la transmission sexuelle des infections entériques, en particulier la shigellose et l'amibiase, peut avoir lieu chez les HARSAH en Colombie-Britannique. D'autres études sont suggérées.

Citation proposée : Narayan S, Galanis E, Groupde de la Colombie-Britannique sur les infections entériques transmises sexuellement. Les infections entériques sont-elles transmises sexuellement en Colombie-Britannique? Relevé des maladies transmissibles au Canada 2016;42-2:27-33. https://doi.org/10.14745/ccdr.v42i02a01f

\author{
Affiliations \\ 'Université Simon Fraser, Burnaby \\ (Colombie-Britannique) \\ ${ }^{2}$ Centre de contrôle des maladies \\ de la Colombie-Britannique, \\ Vancouver (Colombie-Britannique) \\ ${ }^{3}$ Membres du Groupe de la \\ Colombie-Britannique sur les \\ infections entériques transmises \\ sexuellement: Forsting $\mathrm{S}^{4}$, \\ Hoang L ${ }^{2}$, Jeyes $J^{5}$, Nowakowski \\ $\mathrm{C}^{6}$, Ritson $\mathrm{M}^{4}$, Stone $\mathrm{J}^{7}$, Tone $\mathrm{G}^{8}$ \\ ${ }^{4}$ Vancouver Coastal Health \\ Authority, Vancouver \\ (Colombie-Britannique) \\ Interior Health Authority, \\ Kelowna (Colombie-Britannique) \\ ${ }^{6}$ Vancouver Island Health \\ Authority, Victoria \\ (Colombie-Britannique) \\ ${ }^{7}$ Fraser Health Authority, Surrey \\ (Colombie-Britannique) \\ ${ }^{8}$ First Nations Health \\ Authority, Prince George \\ (Colombie-Britannique) \\ *Correspondance : \\ sanaraya@sfu.ca
}

\section{Introduction}

Les entéropathogènes se transmettent le plus souvent en consommant des aliments ou de l'eau contaminés (1-3). Cependant, certains entéropathogènes peuvent aussi se transmettre par des pratiques sexuelles avec un contact fécal-oral, tel qu'une relation sexuelle orale-anale, orale-génitale et anale-génitale (4-6). Même si ces infections entériques transmises sexuellement peuvent se produire chez des personnes hétérosexuelles ayant des relations sexuelles anales non protégées, elles sont plus courantes chez les hommes ayant des relations sexuelles avec d'autres hommes (HARSAH) que chez toute autre population adulte (4,5,7-9).

Les pathogènes qui se transmettent sexuellement incluent I'Entamoeba histolytica, le parasite lamblia, la Shigella $(3,5,7,10-12)$, la Salmonella (13) et la campylobactérie (14). Cependant, I'Entamoeba histolytica, le parasite lamblia et la Shigella sont le plus souvent des infections entériques transmissibles sexuellement $(8,15,16)$. L'ingestion accidentelle de petites quantités de matières fécales contenant seulement 10 à
100 organismes de la bactérie Shigella, de I'Entamoeba histolytica, ou de kystes de parasite lamblia durant des relations sexuelles orales-anales pourrait fournir une dose suffisante pour entraîner une infection. Cette faible dose infectieuse explique également la tendance de ces trois pathogènes à se propager facilement d'une personne à une autre $(7,17,18)$.

La période d'incubation pour la shigellose est courte, de un à deux jours. Elle se caractérise par de la diarrhée (qui peut être sanglante et contenir du pus), de la fièvre et de ténesmes, et est souvent une infection spontanément résolutive (12). Bien que $90 \%$ des infections à l'Entamoeba histolytica soient asymptomatiques, de la fièvre, de la diarrhée et des crampes abdominales peuvent se produire deux à quatre semaines après l'exposition aux parasites. L'infection disparaît avec un traitement en deux semaines (12). La giardiase est généralement asymptomatique chez les humains, mais peut provoquer une faible fièvre, des diarrhées fétides, des crampes abdominales et des ballonnements, une à deux semaines après l'exposition. Les symptômes durent habituellement une à trois semaines et les 
personnes ayant un système immunitaire sain se débarrassent normalement de l'infection toutes seules. Un traitement peut s'avérer nécessaire pour les patients immunovulnérables $(12,19)$.

La shigellose, l'amibiase et la giardiase sont des maladies transmissibles à déclaration obligatoire en

Colombie-Britannique. Même si d'anciens rapports ont souligné des éclosions de shigellose parmi la population des HARSAH en Colombie-Britannique (20-22), I'épidémiologie des infections entériques transmissibles sexuellement, notamment la shigellose, n'a jamais été évaluée en Colombie-Britannique et n'est donc pas bien comprise. L'objectif de cette étude consistait à décrire l'épidémiologie de ces trois infections en

Colombie-Britannique afin de déterminer si une transmission sexuelle peut se produire et de définir la population et les régions présentant un risque d'infection entérale transmissible sexuellement.

\section{Méthodologie}

Une analyse descriptive rétrospective des cas de shigellose, d'amibiase et de giardiase déclarés en

Colombie-Britannique a été réalisée de 2003 à 2012. Tous les cas ont été confirmés en laboratoire et identifiés à partir du Système d'information sur la santé publique intégré (SISP-i). Les renseignements sur l'exposition, notamment l'activité sexuelle et les antécédents de voyage ont été examinés pour tous les cas. Des renseignements de voyage ont été obtenus à partir du Primary Access Regional Information System (PARIS) pour les cas de la Vancouver Coastal Health Authority et à partir du SISP-i pour les autorités sanitaires restantes. Les renseignements de voyage ont été classés dans la catégorie "voyage international » (si le voyage à l'étranger a été signalé dans les quatre jours suivants l'apparition des symptômes) ou « local » (si la personne ne partait pas en voyage, ou voyageait au Canada, en

Tableau 1 : Caractéristiques et taux d'incidence pour 100000 habitants pour les cas déclarés de giardiase, d'amibiase et de shigellose en Colombie-Britannique de 2003 à 2012

\begin{tabular}{|c|c|c|c|}
\hline Caractéristiques & $\begin{array}{l}\text { Giardiase } \\
\text { (Taux d'incidence } \\
\text { [nombre]) }\end{array}$ & $\begin{array}{l}\text { Amibiase } \\
\text { (Taux d'incidence } \\
\text { [nombre]) }\end{array}$ & $\begin{array}{l}\text { Shigellose } \\
\text { (Taux d'incidence } \\
\text { [nombre]) }\end{array}$ \\
\hline Taux d'incidence moyen annuel provincial & $15,2(6593)$ & 7,7 (3 359) & $4,6(1986)$ \\
\hline \multicolumn{4}{|l|}{ Sexe $^{1}$} \\
\hline Femme & $12,1(2648)$ & $4,2(928)$ & $3,7(806)$ \\
\hline Homme & 18,3 (3 933) & $11,2(2422)$ & $5,5(1176)$ \\
\hline Hommes: Femmes & $1,5: 1$ & $2,6: 1$ & $1,5: 1$ \\
\hline \multicolumn{4}{|l|}{ Groupe d'âge (hommes + femmes) } \\
\hline 0 à 9 ans & $26,9(1 \quad 184)$ & $4,1(159)$ & $4,8(200)$ \\
\hline 10 à 19 ans & $10,0(534)$ & $3,9(189)$ & $2,5(131)$ \\
\hline 20 à 59 ans & $16,6(4$ 177) & $11,2(2542)$ & $5,9(1393)$ \\
\hline 60 ans et plus & $7,9(698)$ & $6,1(469)$ & $3,2(262)$ \\
\hline \multicolumn{4}{|l|}{ Autorité sanitaire } \\
\hline Fraser Health Authority & $16,4(2496)$ & $7,8(1$ 199) & $4,6(705)$ \\
\hline Interior Health Authority & $10,7(757)$ & $1,2(89)$ & $1,8(127)$ \\
\hline Northern Health Authority & $9,8(279)$ & $0,2(5)$ & $1,2(34)$ \\
\hline Vancouver Coastal Health Authority & $20,4(2225)$ & $16,2(1768)$ & $8,2(894)$ \\
\hline Island Health Authority & $11,4(836)$ & $4,0(298)$ & $3,1(226)$ \\
\hline \multicolumn{4}{|l|}{ Groupe d'âge des 20 à 59 ans $^{1}$} \\
\hline Femme & $12,6(1592)$ & $5,6(630)$ & $4,6(541)$ \\
\hline Homme & 20,6 (2 578) & $16,7(1905)$ & $7,3(848)$ \\
\hline Rapport hommes-femmes & $1,6: 1$ & $3,0: 1$ & $1,6: 1$ \\
\hline
\end{tabular}

1 Deux cas transgenres non inclus 
Colombie-Britannique ou dans l'autorité sanitaire de résidence) (23). Les renseignements sur l'exposition étaient disponibles uniquement pour la shigellose et l'amibiase, car les cas de giardiase ne font pas l'objet d'un suivi régulier par les autorités de santé publique en Colombie-Britannique. Les renseignements sur les éclosions ont été obtenus à partir du module des sommaires d'éclosions du Réseau canadien de renseignements sur la santé publique, de rapports d'enquête sur les éclosions du Centre de contrôle des maladies de la Colombie-Britannique et de discussions avec des experts.

Les analyses statistiques comprenaient le nombre de cas et les taux d'incidence par année, situation géographique, sexe et groupes d'âge. Les estimations démographiques pour le calcul des taux d'incidence ont été obtenues auprès de BC Stats (disponible à : http://www.bcstats.gov.bc.ca). Les cas ont été regroupés dans l'un des quatre groupes d'âge. Le groupe d'âge des 20 à 59 ans représentait la population sexuellement active, car elle semblait comprendre un nombre excédentaire de cas d'hommes adultes dans l'analyse préliminaire.

Les données ont été analysées à l'aide des logiciels

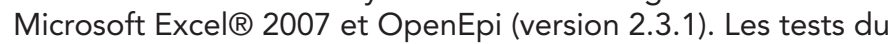
chi carré ont été utilisés pour comparer les proportions de cas de shigellose associés au voyage international par rapport aux cas locaux. Une valeur $p<0,05$ était considérée comme significative. Le risque relatif avec des intervalles de confiance à $95 \%$ a été calculé afin de comparer le risque de shigellose dans ces deux groupes.

\section{Résultats}

Dans l'ensemble, Vancouver Coastal Health Authority a déclaré un taux d'incidence moyen annuel le plus élevé pour les trois infections, suivi par Fraser Health Authority, Island Health Authority, Interior Health Authority et Northern Health Authority (Tableau 1). Un rapport hommes-femmes plus important a été observé pour les trois infections dans toutes les autorités sanitaires; toutefois, la majorité des cas de shigellose et d'amibiase a été déclarée chez les personnes de 20 à 59 ans (5,9 et 11,2 pour 100000 habitants) et pour la giardiase, chez les enfants de 0 à 9 ans (26,9 pour 100000 habitants).

Le taux d'incidence moyen annuel pour la shigellose était de 4,6 pour 100000 habitants (Tableau 1). Le taux d'incidence annuel a fluctué et a atteint un taux maximum en 2003, 2005 et 2007. On a observé le déclin des taux passant de 6,3 en 2007 à 3,8 pour 100000 habitants en 2012 (données non présentées). Globalement, les taux étaient plus élevés chez les hommes que chez les femmes (rapport hommes-femmes de 1,5:1) avec le taux d'incidence le plus élevé chez les hommes de 20 à 59 ans (7,3 pour 100000 habitants) (Figure 1). Les régions de prestation de services de santé de Vancouver ont déclaré le taux moyen annuel le plus élevé à 11,2 pour 100000 habitants (Figure 2).

Quatre éclosions de shigellose ont été signalées entre 2003 et 2012. Les deux premières éclosions ont été provoquées par la Shigella sonnei en 2007 et ont eu lieu dans la région de prestation de services de santé de Vancouver et les régions sanitaires Fraser. La première éclosion a eu lieu au début de 2007 et a touché les HARSAH, et la deuxième éclosion provoquée par une souche différente de Shigella sonnei a eu lieu à la fin de 2007 et a touché les sans-abri (22). Une troisième éclosion de Shigella sonnei associée à un restaurant local a eu lieu en 2010 dans la région de prestation de services de santé de l'Okanagan (20). La dernière éclosion, provoquée par la Shigella flexneri, a eu lieu entre 2008 et 2012 et a touché les HARSAH dans la région de prestation de services de santé de Vancouver (20).

\section{Figure 1 : Répartition des cas de shigellose par catégorie d'âge et par sexe pour la Colombie-Britannique de 2003 à 2012}

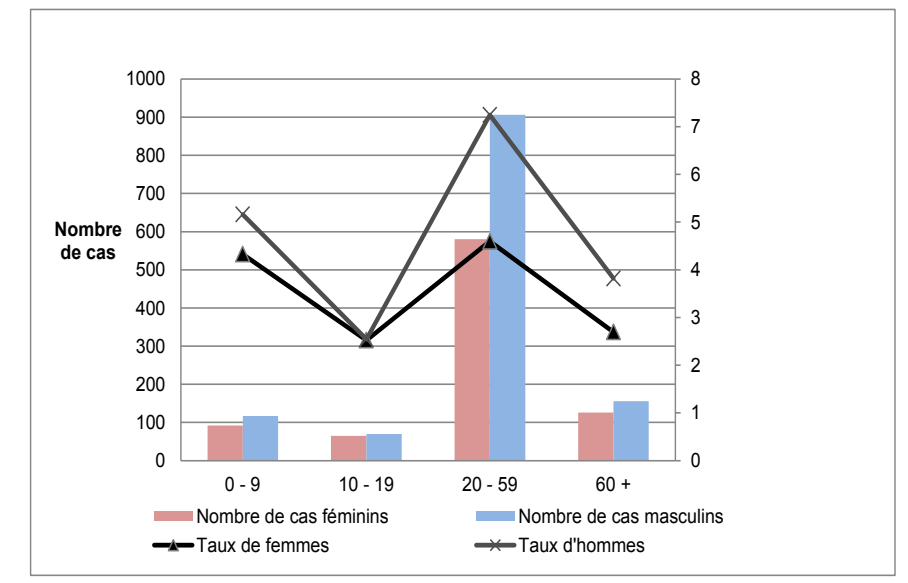

Taux d'incidence moyen annuel de shigellose par région de prestation de service de santé en Colombie-Britannique en 2003 à 2012

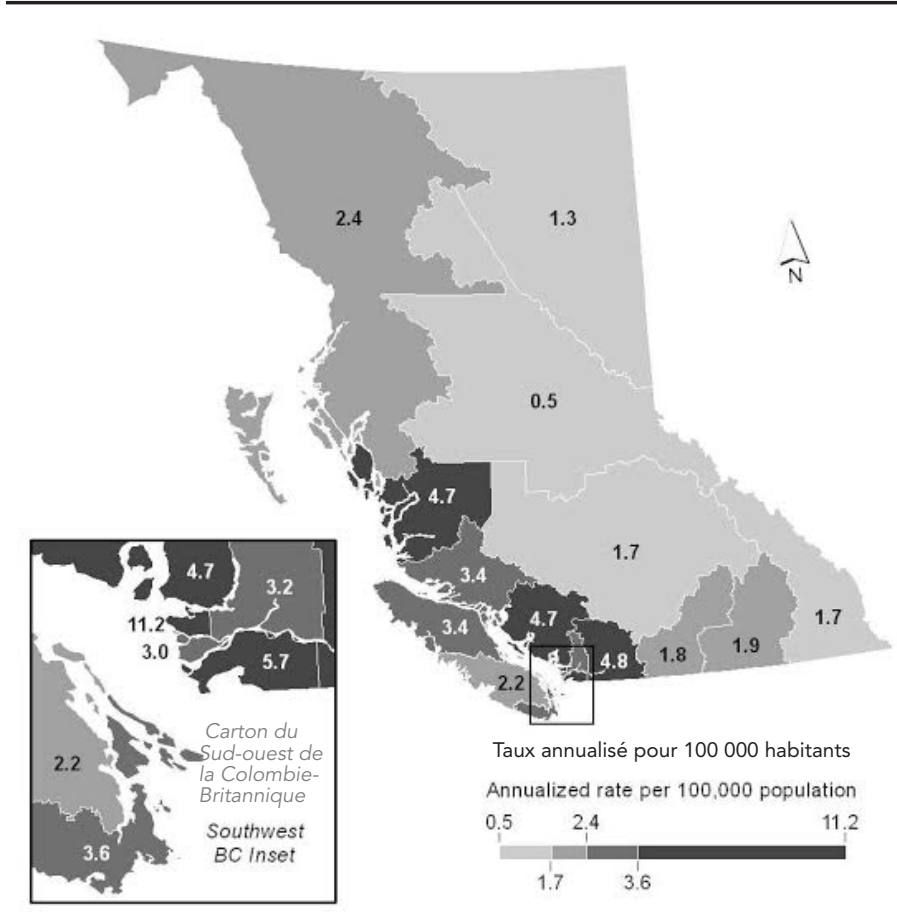


La souche Shigella sonnei $(56,4 \%)$ était la souche la plus souvent signalée en Colombie-Britannique entre 2003 et 2012, suivie par les souches Shigella flexneri $(35,7 \%)$, Shigella boydii $(5,1 \%)$ et Shigella dysenteriae (2,8\%). Les analyses ont révélé un changement de souche Shigella dominante : entre 2003 et 2008, la souche Shigella sonnei était l'espèce infectieuse prévalente (65\%) en Colombie-Britannique et entre 2009 et 2012, la souche Shigella flexneri était l'espèce infectieuse prévalente $(52,1 \%)$.

Les renseignements sur les expositions limitées ont été consignés. Au total, $58 \%$ des cas de shigellose, 15,1\% des cas de giardiase et $8,3 \%$ des cas d'amibiase contenaient des renseignements sur l'exposition dans le SISP-i. Parmi eux, moins de $1 \%$ mentionnait une activité sexuelle en tant qu'exposition. Entre 2008 et 2012, 928 cas de shigellose ont été déclarés aux PARIS et au SISP-i, 654 (70,5 \%) d'entre eux comportaient des renseignements de voyage et 461 cas $(70,4 \%)$ ont été déclarés dans la catégorie "voyage international ". Globalement, les hommes étaient plus à risque de contracter la shigellose localement par rapport aux femmes (RR : 1,6; IC : 1,4 à 1,8). Dans le groupe d'âge des 20 à 59 ans, les hommes avaient un plus grand risque de contracter la shigellose localement par rapport aux femmes (RR : 1,9; IC : 1,7 à 2,4).

Le taux d'incidence moyen annuel pour l'amibiase en Colombie-Britannique était de 7,7 pour 100000 habitants (Tableau 1). Les taux étaient plus élevés chez les hommes que chez les femmes dans tous les groupes d'âge (rapport hommes-femmes de 2,6:1) avec le taux d'incidence le plus élevé chez les hommes de 20 à 59 ans (16,7 pour 100000 habitants). Vancouver Coastal Health Authority a signalé le taux le plus élevé (16,2 pour 100000 habitants). Aucune éclosion n'a été déclarée pendant l'étude.

Le taux d'incidence moyen annuel pour la giardiase en Colombie-Britannique était de 15,2 pour 100000 habitants (Tableau 1). Vancouver Coastal Health Authority a signalé le taux d'incidence moyen annuel le plus élevé $(20,4$ pour 100000 habitants). Le taux d'incidence était plus élevé chez les hommes que chez les femmes dans tous les groupes d'âge (rapport hommes : femmes de 1,5:1), avec le taux d'incidence le plus élevé observé chez les enfants de sexe masculin âgés de 0 à 9 ans (28,9 pour 100000 habitants) (données non présentées). Aucune éclosion n'a été déclarée pendant l'étude.

\section{Discussion}

Les résultats de cette étude révèlent des taux plus élevés des trois infections dans la région Vancouver Coastal Health, avec un rapport hommes-femmes plus élevé. Cependant, le taux d'incidence était le plus élevé chez les hommes adultes pour la shigellose et l'amibiase et chez les enfants (0 à 9 ans) pour la giardiase. Les taux plus élevés chez les adultes (pour la shigellose et l'amibiase) peuvent être dus en partie à une transmission sexuelle.

Pour la shigellose et l'amibiase, il y avait un rapport élevé hommes-femmes et un taux d'infection plus élevé chez les hommes âgés de 20 à 59 ans, par rapport à tous les autres groupes d'âge et de sexe. De plus, pour la shigellose, les hommes adultes étaient significativement plus susceptibles que les femmes de contracter la shigellose localement et un nombre inquiétant de cas de shigellose chez les hommes adultes a été observé dans la région de prestation de services de santé de Vancouver (données non présentées). En outre, deux des quatre éclosions de shigellose déclarées en Colombie-Britannique concernaient les HARSAH. Dans ces deux éclosions, aucun autre facteur de risque commun tel que l'alimentation, les restaurants ou le voyage n'a été observé et l'on pense que les pratiques sexuelles (sexe oral-anal) étaient le mode de transmission. Cela est contraire à la giardiase qui concernait les hommes plus que les femmes dans tous les groupes d'âge avec le taux le plus élevé signalé chez les enfants de sexe masculin âgés de 0 à 9 ans et qui indique une transmission sexuelle moins probable.

Au Canada, d'autres éclosions de shigellose dans lesquelles la transmission sexuelle était la cause ont été déclarées. Entre 1999 et 2001, une éclosion de $S$. sonnei et de $S$. flexneri a touché les HARSAH au Québec $(24,25)$ et en juillet 2014, un groupe de cas de shigellose a été déclaré chez les HARSAH à Toronto. De nombreux pays développés signalent des éclosions de shigellose touchant les HARSAH (26-33). Chez les HARSAH, la shigellose est surtout une infection transmise sexuellement (15), et le plus grand risque de transmission est associé aux pratiques sexuelles ayant un contact oral-anal direct $(7,8,15,25,27,31)$. De plus, le fait d'avoir plusieurs partenaires sexuels pourrait être la raison de la vaste dissémination de la Shigella chez les HARSAH (34). On a aussi déterminé que l'infection par le virus de l'immunodéficience humaine était également un facteur de risque important pour la shigellose chez les $\operatorname{HARSAH}(7,15,27)$.

L'analyse a démontré un changement de l'espèce infectieuse de Shigella dominante (Shigella sonnei à Shigella flexneri) vers l'année 2009. Wilmer et al. (20) ont signalé des trouvailles semblables dans une étude dans laquelle la Shigella flexneri est devenue la souche circulante dominante chez les HARSAH dans le centre de la ville de Vancouver après 2008. D'autres personnes ont également signalé un changement d'espèce Shigella dominante chez la même population de HARSAH $(27,35,36)$. Ces changements peuvent refléter un certain degré d'immunité collective à une espèce de Shigella donnée (7).

Cette étude a également révélé que les hommes adultes étaient plus susceptibles que les femmes adultes de contracter la shigellose localement $(R R=1,9)$. Une constatation semblable a été signalée dans une éclosion de shigellose au Pays de Galle, où la shigellose contractée localement était prédominante chez les hommes ayant signalé une activité HARSAH la semaine précédant la maladie (33). Un plus grand risque de voyage chez les femmes est un indicateur de contraction de la shigellose par des facteurs de risque considérés comme étant plus courants durant les voyages, notamment les aliments et l'eau contaminés.

L'incidence moyenne annuelle la plus élevée pour l'amibiase a été signalée chez les hommes adultes

(16,7 pour 100000 habitants). Les raisons ne sont pas claires. Il y a avait très peu de renseignements relatifs à l'exposition sexuelle sur l'amibiase. L'Entamoeba histolytica se transmet généralement d'une personne à une autre (10) ou par de l'eau contaminée (12). Étant donné que la plupart des habitants 
de la Colombie-Britannique ont accès à de l'eau potable (37), à l'exception du voyage dans des pays endémiques, la transmission sexuelle semble probable pour ce groupe d'âge et de sexe. La transmission sexuelle de l'E. histolytica a été signalée chez les HARSAH avec des pratiques sexuelles orales-anales considérées comme étant le mode de transmission $(16,38,39)$.

Le taux de giardiase était plus élevé pour les hommes dans tous les groupes d'âge, et les enfants de sexe masculin âgés de 0 à 9 ans avaient le taux le plus élevé. Cette constatation est semblable aux observations signalées par d'autres pays développés (40-43) où la giardiase infectait le plus souvent de petits enfants dans les garderies et la transmission était associée à une mauvaise hygiène des mains. Avec un schéma semblable de giardiase en Colombie-Britannique, la transmission sexuelle de la Giardia semble moins probable, ou peut avoir lieu à de faibles taux, ou est submergée par d'autres voies de transmission telles que le contact avec de l'eau contaminée, le voyage vers des pays endémiques et la transmission dans des garderies (40).

Bien que cette étude ait démontré une incidence plus importante de certaines infections entériques chez les hommes de 20 à 59 ans, tous les nombres inquiétants de maladies chez tous les hommes adultes ne peut pas être attribué à la transmission sexuelle et toutes les transmissions sexuelles ne seront pas observables en nombre inquiétant chez les hommes adultes. La combinaison de l'âge adulte et du sexe masculin utilisée pour déterminer une population vulnérable n'est pas un indicateur propre à la transmission sexuelle. D'autres facteurs (tels que l'eau contaminée, l'exposition au travail et récréative en plein-air) peuvent aussi être à l'origine de la plus grande incidence observée dans ce groupe. En outre, les adultes hétérosexuels sont également à risque d'infection entérale transmissible sexuellement par contact oral-anal. Des renseignements manquants sur l'exposition en raison du manque d'entrevues des cas, une évaluation incomplète des facteurs de risque sexuels ou des saisies de données incomplètes nuisent encore plus à l'attribution des cas à une voie de transmission précise.

Malgré la littérature croissante sur le risque des infections entérales transmissibles sexuellement, des lignes directrices sur la prévention et des renseignements pédagogiques pour les populations vulnérables ne sont pas disponibles à grande échelle. Les lignes directrices de santé publique à ce sujet ne semblent pas être disponibles au Canada. Des telles lignes directrices pourraient contribuer à l'enquête nécessaire en matière de santé publique pour mieux évaluer le risque d'infections entérales transmissibles sexuellement et les modes de transmission, le besoin de recherche de contacts et de messages de sensibilisation recommandés pour des cas, les populations vulnérables et la population générale. Les conclusions de cette étude ont mené à un examen des formulaires de suivi des cas entériques provinciaux dans le but de mieux recueillir les renseignements relatifs aux pratiques sexuelles qui augmentent le risque d'infection entérale transmissible sexuellement.

Un diagnostic et un traitement en temps opportun des infections entériques réduiront la durée de la maladie et permettront d'interrompre sa transmission (4, 29). Actuellement, les Lignes directrices canadiennes sur les infections transmissibles sexuellement recommandent aux fournisseurs de soins de santé d'effectuer un dépistage d'entéropathogènes si les clients signalent des activités sexuelles anorectales ou présentent des symptômes compatibles (4). En outre, les fournisseurs de soins de santé doivent donner des conseils sur les relations sexuelles sécuritaires en fonction d'une évaluation personnalisée des risques de santé en matière de sexualité (29). Actuellement, d'importants efforts visant à sensibiliser les populations vulnérables ont été signalés durant les éclosions d'infections entérales transmises sexuellement $(27,44)$. Cependant, pour améliorer la sensibilisation sur les infections entérales transmises sexuellement chez les populations vulnérables, les messages de promotion de la santé en matière de sexualité doivent avoir lieu de façon plus sécuritaire et doivent contenir des renseignements sur les infections entérales transmises sexuellement, des conseils pour éviter le contact oral-anal non protégé (surtout si le partenaire est malade), l'hygiène des mains à la suite d'un contact sexuel, et doivent demander un avis médical pour la gastroentérite $(15,31)$.

\section{Conclusion}

Cette étude laisse entendre que la transmission sexuelle des infections entériques, en particulier la shigellose et I'amibiase, peut avoir lieu chez les HARSAH à Vancouver (Colombie-Britannique). Cette conclusion est appuyée par des données sur les éclosions et un historique des expositions (activité sexuelle, voyage) limitées des cas. Pour réduire I'incidence des infections entérales transmises sexuellement, les interventions de santé publique doivent aller plus loin que la salubrité des aliments et les pratiques d'hygiène des mains. Une surveillance continue, en particulier de l'historique d'exposition des cas, peut également contribuer à guider les interventions de santé publique afin de réduire les infections entérales transmises sexuellement.

\section{Remerciements}

Les auteurs remercient les autorités sanitaires régionales et les agents d'hygiène du milieu engagés dans la collecte de données de surveillance et les suivis des cas, et les laboratoires de la Colombie-Britannique engagés dans le diagnostic des cas inclus dans l'article. Nous aimerions également remercier Marsha Taylor, Michael Otterstatter, Sophie Li et surtout Dr Pablo Nepomnaschy pour leur orientation et leurs commentaires précieux durant ce projet.

\section{Conflit d'intérêts}

Aucun.

\section{Financement}

Aucun. 


\section{Références}

1. Fletcher SM, Damien S, Harkness J, Ellis J. Enteric Protozoa in the developed world: A public health perspective. Clin Microbiol Rev. July 2012;25(3):420-449.

2. Public Health Agency of Canada. Overview: FoodNet Canada: Reducing the burden of gastrointestinal disease in Canada. Ottawa ON: Public Health Agency of Canada; 2013. http://www.phac-aspc.gc.ca/foodnetcanada/overviewapercu-eng.php.

3. Centers for Disease Control and Prevention (CDC). Division of Foodborne, Waterborne, and Environmental Diseases: Enteric Diseases Epidemiology Branch . Washington DC: CDC; 2013. http://www.cdc.gov/ncezid/dfwed/edeb/.

4. Public Health Agency of Canada. Canadian guidelines on sexually transmitted infections. Ottawa ON: Public Health Agency of Canada; 2013. (Disponible en français : http:// www.phac-aspc.gc.ca/std-mts/sti-its/cgsti-ldcits/section-4-6fra.php).

5. Wiwanitkit V. Sexually transmitted Shigellosis. Sex Disab. 2006;24(1).

6. Rompalo AM. Diagnosis and treatment of sexually acquired proctitis and proctocolitis: An update. Clin Infec Dis. 1998;28(Supplement 1):S84-S90 .

7. Daskalakis DC, Blaser MJ. Another perfect storm: Shigella men who have sex with men, and HIV. Clin Infec Dis. 2007;44(3):335-337.

8. Tauxe RV, Macdonald RC, Hargrett-Bean N, Blake PA. The persistence of Shigella flexneri in the United States: Increasing role of adult males. Am J Public Health. Nov 1998;78(11):1432-1435.

9. Mildvan D, Gelb AM, William D. Venereal transmission of enteric pathogens in male homosexuals: Two case reports. JAMA. 1977;238(13):1387-1389.

10. Ortega HB, Borchardt KA, Hamilton R, Ortega P, Mahood J. Enteric pathogenic protozoa in homosexual men from San Francisco. Sex Transm Dis. Apr - Jun 1984;11(2):59-63.

11. Tessier J, Gal D. Giardiasis. Prim Care Update. 1999;6(1):1-4.

12. Public Health Agency of Canada. Pathogen safety data sheet; Infectious Substances. Ottawa ON: Public Health Agency of Canada; 2011. (Disponible en français : http:// www.phac-aspc.gc.ca/lab-bio/res/psds-ftss/index-fra.php).

13. Reller ME, Olsen SJ, Kressel AB, Moon TD, Kubota KA, Adcock MP, Mintz ED. Sexual transmission of typhoid fever: A multistate outbreak among men who have sex with men. Clin Infec Dis. 2003;37(1):141-144.

14. Quinn TC, Goodell SE, Fennell C, Wang SP, Schuffler MD, Holmes KK, Stamm WE. Infections with Campylobacter jejuni and Campylobacter-like organisms in homosexual men. Ann Intern Med. Aug 1984;101:187-192.

15. Aragón TJ, Vugia D, Shallow S, Samuel MC, Reingold A, Agngulo FJ, Bradford WZ. Case-control study of Shigellosis in San Francisco: The role of sexual transmission and HIV infection. Clin Infec Dis. 2007;44(3):327-334.
16. Stark D, Van Hal SJ, Mathews G, Harkness J, Marriott D. Invasive amebiasis in men who have sex with men, Australia. Emerg Infec Dis, Jul 2008;14(7):1141-1143.

17. DuPont HL, Levine MM, Hornick RB, Formal SB. Inoculum size in shigellosis and implications for expected mode of transmission. J Infect Dis, June 1989;159(6):1126-8.

18. Klausner J, Hook E. Current diagnosis and treatment of sexually transmitted diseases. New York: McGraw-Hill Medical; 2007.

19. BC Centre for Disease Control. Diseases and conditions: Giardiasis overview . Vancouver BC; BCCDC; 2010. http:// www.bccdc.ca/health-info/diseases-conditions/Giardiasis.

20. Wilmer A, Romney MG, Gustafson R, Sandhu J, Chu T, Ng C, Hull MW. Shigella flexneri Serotype I in men who have sex with men in Vancouver, Canada. HIV Med. 2015;16(3):168175.

21. Strauss B, Kurzac C, Embree G, Sevigny R, Paccagnella A, Fyfe M. Clusters of Shigella sonnei in men who have sex with men, British Columbia, 2001. Can Comm Dis Rep. Jul 2001;27(13):109-10.

22. Canadian Network for Public Health Intelligence (CNPHI). Outbreak module. Ottawa ON: CNPHI; 2014. https://www. cnphi-rcrsp.ca.

23. Taylor M, MacDougall L, Li M, Galanis E, BE. The impact of international travel on the epidemiology of enteric infections, British Columbia, 2008. Can J Public Health. 2010;101(4):332-36.

24. Public Health Agency of Canada. Canadian integrated surveillance report: Salmonella, Campylobacter, verotoxigenic E. coli and Shigella, from 2000 to 2004. Can Comm Dis Rep. December 2009;35S3: 1-50. (Disponible en français : http://www.phac-aspc.gc.ca/publicat/ccdrrmtc/09vol35/35s3/shigella-shigella-fra.php).

25. Gaudreau C, Bruneau A, Ismaïl J. Outbreak of Shigella flexneri and Shigella sonnei enterocolitis in men who have sex with men, Quebec, 1999 to 2001. Can Comm Dis Rep. April 2005;31(8). (Disponible en français : http://www.phacaspc.gc.ca/publicat/ccdr-rmtc/05vol31/rm3108a-fra.php)

26. Morgan O, Crook P, Cheasty T, Jiggle B, Giraudon I, Hughes $H$. Shigella sonnei outbreak among men who have sex with men - San Francisco, California, 2000-2001. MMWR. Oct 2001;50(42):922-6.

27. Morgan $O$, Crook P, Cheasty T, Jiggle B, Giraudon I, Hughes $\mathrm{H}$, Jones SM. Shigella sonnei outbreak among homosexual men, London. Emerg Infec Dis. Sept 2006;12(9):1458-1460.

28. O'Sullivan B, Valerie D, Pontivivo G, Karagiannis T, Marriott D, Harkness J. Shigellosis linked to sex venues, Australia. Emerg Infec Dis. Aug 2002;8(8):862-864.

29. Rowe SL, Radwan S, Lalor K, Valcanis M, Gregory JE. An outbreak of shigellosis among men who have sex with men, Victoria, 2008. Vic Inf Dis Bull. Dec 2010;13(4):119-121.

30. Marcus U, Zucs P, Bremer V, Osamah H. Cluster of shigellosis in men in Berlin in 2001. Euro Surveill. 2002;6(33):1862. 
31. Marcus U, Zucs P, Bremer B, Hamouda O, Prager R, Tschaepe $H$, Kramer M. Shigellosis: A re-emerging sexually transmitted infection: outbreak in men having sex with men in Berlin. Int J STD AIDS. Aug 2004;15(8):533-537.

32. Okame M, Adachi E, Sato H, Shimizu S, Kikuchi T, Miyazaki N, Koga M. Shigella sonnei outbreak among men who have sex with men in Tokyo. Jpn J Infect Dis. 2012;65:277-278.

33. Borg ML, Modi A, Tostmann A., Gobin M, Cartwright J, Quigley C. Ongoing outbreak of Shigella flexneri serotype $3 a$ in men who have sex with men in England and Wales, data from 2009-2011. Euro Surveill. 2012;17(13):20137.

34. Drusin LM, Genvert G, Topf-Olstein B, Levy-Zombek E. Shigellosis. Another sexually transmitted disease? Sex Transm Infec. Oct 1976;52(5):348-350.

35. Ratnayake R, Allard R, Pilon PA. Shifting dominance of Shigella species in men who have sex with men. Epidemiol Infect. 2012;140(11):2082-2086.

36. Pro-MED mail database. Shigellosis, changing epidemiology - Canada. Brookline MA: International Society for Infectious Diseases: Feb 16 2010. http://www.promedmail.org/ post/383254.

37. Office of the Provincial Health Officer. Progress on the Action Plan for Safe Drinking Water in BC, 2011. Victoria BC: Ministry of Health; 2012. http://www.health.gov.bc.ca/pho/ pdf/drinking-water-report-2011.pdf

38. Ohnishi K, Kato Y, Imamura A, Fukayama M, Sunoda T, Sakaue Y, Sagara H. Present characteristics of symptomatic Entamoeba histolytica infection in the big cities of Japan. Epidemiol Infect. Jan 2004;132(1):57-60.

39. Zhou F, Li M, Yang Y, Gao C, Li X, Jen Q, Gao L. Seroprevalence of Entamoeba histolytica infection among Chinese men who have sex with men. Negl Trop Dis. May 2013;7(5):e2232.

40. Espelage W, Heiden MA, Stark K, Alpers K. Characteristics and risk factors for symptomatic Giardia lamblia infections in Germany. BioMed Cent Public Health. Jan 2010;10(41).

41. Gray SF, Gunnell DJ, Peters TJ. Risk factors for Giardiasis: A case-control study in Avon and Somerset. Epidemiol Infect. August 1994;113(1):95-102.

42. Laupland KB, Church DL. Population-based laboratory surveillance for Giardia sp. and Cryptosporidium sp. infections in a large Canadian health region. BioMed Cent Infect Dis. Sept 2005;5(72).

43. Yoder JS, Beach MJ. Giardiasis surveillance: United States, 2003-2005. MMWR Surveill Summ. Sept 2007;56(SS07):1118.

44. Klausner JD, Aragon T, Enanoria WT, Mann JK, Zapitz VM, Portnoy D. Shigella sonnei outbreak among men who have sex with men: San Francisco, California. 2000-2001. MMWR. 2001;50(42):922-926. 